\title{
Combination with allogenic bone reduces early absorption of $\beta$-tricalcium phosphate ( $\beta$-TCP) and enhances the role as a bone regeneration scaffold. Experimental animal study in rat mandibular bone defects
}

Makoto HIROTA, Yoshiro MATSUI, Nobuyuki MIZUKI, Teruki KISHI, Kei WATANUKI, Tomomichi OZAWA, Takafumi FUKUI, Shihomi SHOJI, Makoto ADACHI, Yuka MONDEN, Toshinori IWAI and Iwai TOHNAI

Department of Oral and Maxillofacial Surgery, Yokohama City University Graduate School of Medicine, 3-9 Fuku-ura, Kanazawa-ku, Yokohama 236-0004, Japan

Corresponding author, Makoto HIROTA; E-mail: mhirota@med.yokohama-cu.ac.jp

$\beta$-tricalcium phosphate ( $\beta$-TCP) was grafted into rat mandibular bone defects to assess its potential as a scaffold material for bone regeneration. For this purpose, $\beta$-TCP (TCP), allogenic bone (Allograft), and allogenic bone combined with $\beta$-TCP (Combined) were employed as graft materials. To the left side of the graft materials in the bone defects, platelet-rich plasma (PRP) was added. The rats were sacrificed at one, three, and five weeks. Bone formation rate (BFR), remaining $\beta$ TCP rate (RTR), $\beta$-TCP absorption rate (TAR), whole amount of $\beta$-TCP (WTCP), and total rate of BFR and RTR (TBR) were measured. Combined showed equivalent BFR to Allograft at five weeks, and showed higher RTR at one week and higher BFR at five weeks than TCP. Combined with PRP showed higher TAR than that without PRP at three weeks. Therefore, combination with allogenic bone showed reduced $\beta$-TCP absorption, hence enhancing the role of $\beta$-TCP in bone regeneration. These findings suggested that $\beta$-TCP is a better scaffold for bone regeneration if its early absorption is reduced when used in combination with an osteogenic material.

Key words: $\beta$-TCP, Scaffold, Bone regeneration

Received May 8, 2007: Accepted Jun 27, 2008

\section{INTRODUCTION}

Many graft materials have been used for the repair, restoration, and reconstruction of bone defects in oral and maxillofacial surgery. Among the wide range of bone graft materials, autogenous bone is widely accepted as the gold standard for graft material. Autogenous bone, however, needs to be harvested from other sites of the body such as the iliac crest and tibia. Recently, to reduce or replace the harvesting of autogenous bone, various kinds of allografts and alloplasts - natural or synthetic bone substitutes such as freeze-dried bone allograft (FDBA), demineralized FDBA (DFDBA), bovinederived porous bone material, and synthetic calcium phosphate - have been developed.

$\beta$-tricalcium phosphate ( $\beta$-TCP), a synthetic calcium phosphate ceramic, has recently been utilized as a bone substitute to repair bone defects ${ }^{1}$. $\quad \beta$-TCP is a biocompatible and absorbable material, and the mechanism of bone formation in $\beta$-TCP is osteoconduction. Using this material, newly formed bone forms along the surface.

However, $\beta$-TCP is not without shortcomings. Drawbacks associated with $\beta$-TCP include early absorption independent of bone formation and hence resulting in insufficient bone replacement, and that it also induces ectopic bone formation due to residues remaining in regenerated bone tissue ${ }^{2,3)}$. These problems could be related to the degradability of this material, and the rate of degradability is determined by the microporosity of $\beta$-TCP ${ }^{4}$. To overcome this problem, highly pure $\beta$-TCP was developed. Recently, in oral and maxillofacial surgery, highly pure $\beta$-TCP has been utilized in bone reconstruction as a scaffold for enhanced bone regeneration ${ }^{5-7}$.

In the present study, the objective was to assess the potential of highly pure, porous $\beta$-TCP granules, OSferion $^{\circledR}$ (Olympus Biomaterial Corp., Tokyo, Japan), as a scaffold for bone regeneration. One contributing reason for using OSferion ${ }^{\circledR}$ in this study was that it has been reported that OSferion ${ }^{\circledR}$ was absorbed with favorable bone formation ${ }^{8)}$. Rat mandibular bone defects were thus grafted with OSferion ${ }^{\circledR}$ with or without allogenic bone, and then the bone formation and absorption rates were evaluated. In addition, platelet-rich plasma (PRP) was added to these graft materials within the bone defects to evaluate its efficacy. The rats were sacrificed at one, three, and five weeks after surgery. This paper thus discusses the capability, degradability, and compatibility of the $\beta$-TCP granules as a bone regeneration scaffold in combination with other graft materials. 


\section{MATERIALS AND METHODS}

\section{$\beta-T C P$}

OSferion ${ }^{\circledR}$ (G1 Type, Olympus Terumo Biomaterial Corp., Tokyo, Japan), highly pure porous $\beta$-TCP granules, was used in the present study. Fine $\beta$ TCP powder was synthesized by wet milling (a mechanochemical method). A slurry mixture of $\mathrm{CaCO} 3$ and $\mathrm{CaHPO} 4 \cdot 2 \mathrm{H} 2 \mathrm{O}$ at a molar ratio of $1: 2$ with pure water was prepared in a mill pot for 24 hours, and then dried at $80^{\circ} \mathrm{C}$. Calcium-deficient hydroxyapatite was obtained, and the crystal was converted to $\beta$ TCP by calcination at $750^{\circ} \mathrm{C}$. $\beta$-TCP was mixed to obtain a foaming slurry, which was dried at room temperature for one day and at $40^{\circ} \mathrm{C}$ for another day. By sintering the material at $1050^{\circ} \mathrm{C}, \quad \beta$-TCP exceeding $99.9 \%$ purity was obtained. Porosity was $75 \%$ and pore size ranged from 100 to $400 \mu \mathrm{m}$ with interconnectivity. Subsequently, $\beta$-TCP was shaped into a granular form with a diameter of 250-500 $\mu \mathrm{m}$. Figure 1 shows a scanning electron micrograph (SEM) of the porous $\beta$-TCP thus obtained in this study.

\section{PRP preparation}

Whole blood drawn from rats was combined with anticoagulant citrate dextrose phosphate (ACD-A). The blood was centrifuged at 2,400 rpm for 10 minutes to separate the plasma. After which, the plasma was drawn off and centrifuged at 3,600 rpm for 15 minutes to separate the platelets. Plateletpoor plasma (PPP) was separated from PRP, and only PRP was used in this study. PRP was activated at bone graft surgery with calcium chloride solution and bovine thrombin, and used with or without graft materials. When used with graft materials, PRP was mixed well with the materials. The platelet count in PRP drawn from rats was $2.15 \times 10^{6} / \mathrm{mm}^{3}$.

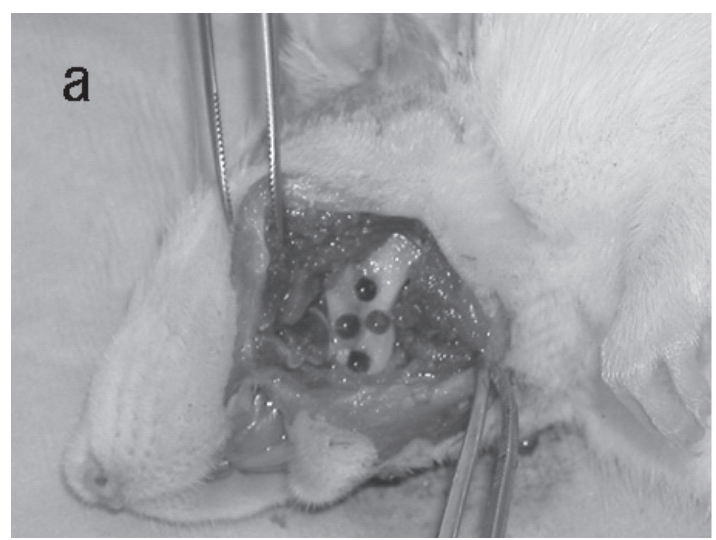

Fig. 2 Surgical sites of rat mandibular bone defects before (a) and after (b) bone graft surgery.

Mixture of allogenic bone and $\beta$-TCP granules (Combined) $\left(^{*}\right), \beta$-TCP granules alone (TCP) $(* *)$, allogenic bone alone (Allograft) $(* * *)$, and control site $(* * * *)$.

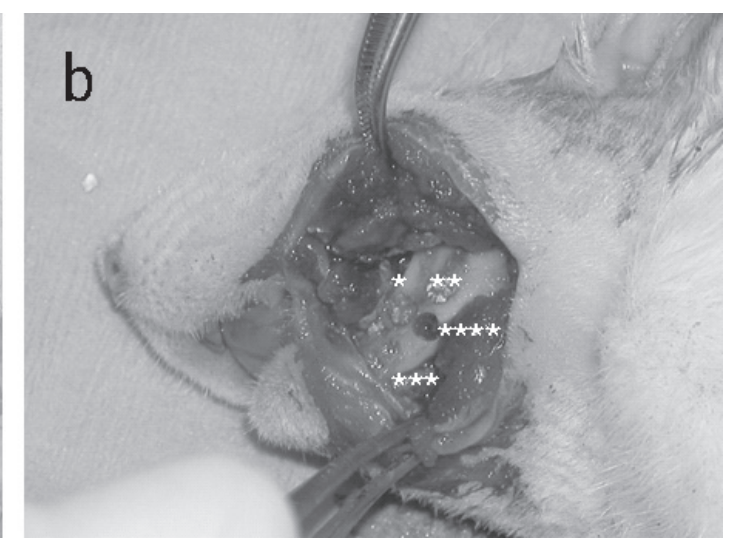

\section{Bone graft surgery}

Seven-week-old male Wistar rats between 150 and $200 \mathrm{mg}$ body weight, purchased from Charles River Japan (Yokohama, Japan), were used. To evaluate the efficacy of $\beta$-TCP compared with allogenic materials, allogenic bone grafting was performed.

The National Guidelines for the Care and Use of Laboratory Animals were observed during and after the bone graft surgery. First, allogenic bone was harvested from the rat's thigh bone. Then, graft surgery was performed under a general anesthesia with an intraperitoneal injection of pentobarbital at a dose of $50 \mathrm{mg} / \mathrm{kg}$ body weight.An incision was made parallel to the inferior border of the mandible and the periosteum of the mandible was reflected. After the periosteum was incised and elevated, four defects of $2-\mathrm{mm}$ diameter and depth were created in each side of the mandible with a round bur under copious irrigation (Fig. 2a). Three of these bone defects were

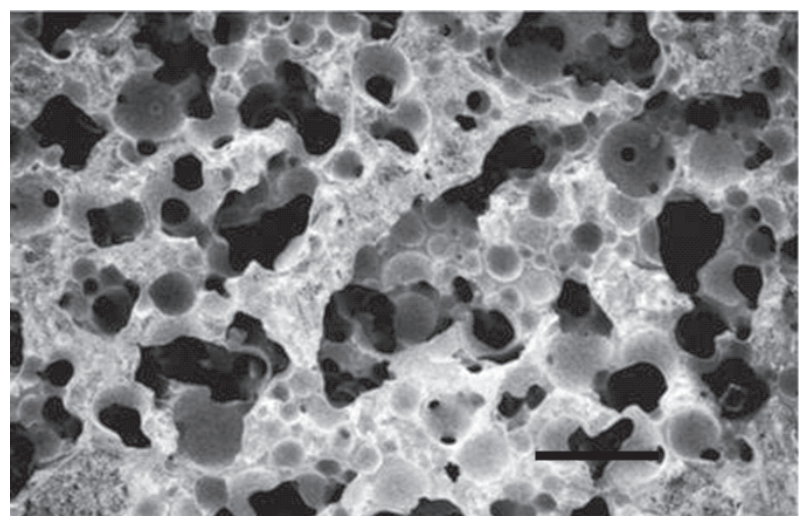

Fig. 1 Scanning electron micrograph (SEM) of highly pure porous $\beta$-TCP. Black scale bar shows $5 \mu \mathrm{m}$. Various sizes of micropores are seen. (Photograph courtesy of Olympus Terumo Biomaterial Corp.)
(**), allogenic bone alone (Allograft) (***), and control site $(* * * *)$. 
either filled with allogenic bone (Allograft), $\beta$-TCP granules (TCP), or allogenic bone combined with $\beta$ TCP granules (Combined). As a control, no material was grafted into the fourth bone defect (Fig. 2b). For the Combined graft material, allogenic bone and $\beta$ TCP granules were mixed at a ratio of 50:50 volume. As for the prepared PRP, it was added to these graft materials only on the left side within the bone defect. The wound was closed with 5-0 nylon sutures.

\section{Bone samples and statistical analysis}

The rats were sacrificed with an intraperitoneal overdose of sodium pentobarbital at one, three, and five weeks after surgery. There were three rats in the one-week group and four rats in the three- and five-week groups. The mandibular bone was removed and bone formation was histologically evaluated. Bone samples were fixed in $4 \%$ formalin; perfusionfix of these rats was not performed.

To obtain undecalcified bone sections, the bone samples were rinsed, dehydrated, and embedded in methyl methacrylate (MMA). After cutting into sections of $10 \mu \mathrm{m}$ thickness, these specimens were stained with toluidine blue.

All areas of newly formed bone (NB) and remaining $\beta$-TCP (RT), detected in a half-circle area on each specimen, were calculated with an NIH Image analysis program (National Institute of Health, Bethesda, MD). The measurement method is shown in Fig 3 . The ratios of the newly formed bone area $(\mathrm{NB})$ and the remaining $\beta$-TCP area $(\mathrm{RT})$ per measurement area (MA) on the specimens were defined as the bone formation rate (BFR) (\%) and remaining $\beta$-TCP rate (RTR) (\%) respectively. From RTR, the $\beta$-TCP absorption rate (TAR) (\%) was calculated. As the total volume of $\beta$-TCP granules was different between the TCP and Combined groups, the amount of remaining $\beta$-TCP was doubled in the RTR calculation for the Combined group. Thereafter, the total rate of BFR and RTR (TBR) was calculated. Moreover, an estimate of the whole amount of $\beta$-TCP (WTCP) in each week was calculated from the remaining $\beta$-TCP amount on specimens and the amount of $\beta$-TCP at surgery. WTCP at surgery was considered as $100 \%$ in the TCP group and 50\% in the Combined group respectively.

\section{Statistical analysis}

BFR, RTR, and TAR of each group were analyzed using Tukey-Kramer's test. Specimens' means were considered significantly different if $\mathrm{P}$ values were less than 0.05. In addition, for Combined and TCP groups that used $\beta$-TCP granules, TBR and WTCP were also analyzed and compared between these groups using Tukey-Kramer's test.

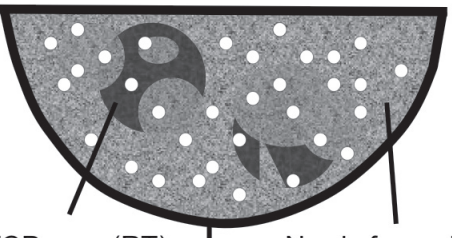

Rest $\beta$-TCP area (RT) $\quad$ Newly formed bone area (NB)

Measurement area (MA)

BFR; bone formation rate in all groups = NB / MA x $100(\%)$

RTR; rest $\beta-\mathrm{TCP}$ rate in TCP $=\mathrm{RT} / \mathrm{MA} \times 100(\%)$

${ }^{*}$ RTR; rest $\beta$-TCP rate in Combined $=$ RT $\times 2$ / MA $\times 100(\%)$

TAR; $\beta$-TCP absorption rate in TCP, Combined $=100-$ RTR (\%)

TBR; Total rate of BRF and RTR in TCP, Combined = BFR + RTR (\%)

WTCP; Whole amount TCP in TCP, Combined = RT / MA x $100(\%)$

Fig. 3 Schema of measurement method and abbreviations used in the present study. The measurement area (dark solid line), remnant $\beta$-TCP area (RT, dark gray), and newly formed bone area (NB, light gray) on the specimen model are shown. From the measurement of these areas, BFR, RTR, TAR, TBR, and WTCP are calculated.

*: In the Combined group, the amount of $\beta$-TCP at surgery was half of that in TCP group. As such, RT was doubled to calculate RTR.

\section{RESULTS}

\section{Histological findings}

At one week, bone formation induced by the graft materials was not sufficient (Fig. 4). Remaining $\beta$ TCP was seen in both TCP and Combined groups, whereby approximately $30 \%$ of $\beta$-TCP remained in TCP (Fig. 4c). Further, the amounts of remaining $\beta$ TCP in the measurement areas were equivalent between the TCP and Combined groups (Figs. 4c, d).

In the Combined-with-PRP group, bone growth into the micropores of $\beta$-TCP was seen (Fig. $4 \mathrm{~d}$ ), while there was clearly no new bone formation in the micropores for TCP (Fig. 4c).

At three weeks, the control site (Fig. 5a) showed newly formed, thin trabeculae forming a loose continuous network and that the connection between new bone and original bone was weak. In Allograft group without PRP (Fig. 5b), the newly formed bone consisted of woven bone with rigid connection between the new bone and original bone. In TCP group without PRP (Fig. 5c), bone growth into the micropores of remaining $\beta$-TCP could be seen but there was insufficient newly formed bone. In Combined group without PRP (Fig. 5d), the defect area was uniformly filled with newly formed bone 

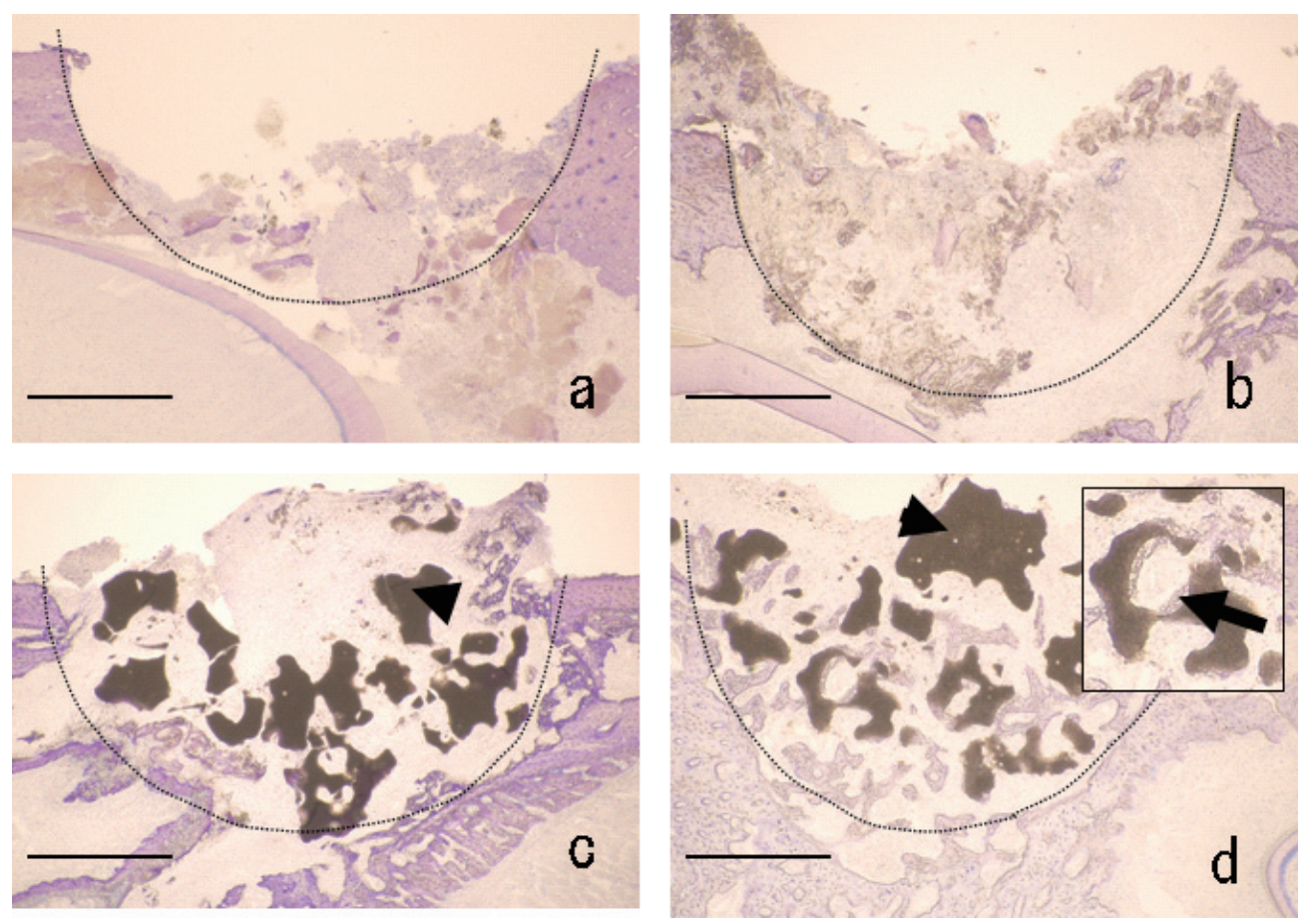

Fig. 4 Histological findings of graft materials $(b-d)$ and control site (a) with PRP in rat mandibular bone defects at one week after graft surgery. Measurement area is shown as a dotted line. (a) Control and (b) Allograft groups with PRP: a little newly formed bone is seen. (c) TCP with PRP: remnant $\beta$-TCP is seen (arrowhead). Newly formed bone around $\beta$-TCP is poor. (d) Combined-with-PRP group: remnant $\beta$-TCP is seen (arrowhead). Newly formed bone around $\beta$-TCP and into micropores is seen (arrow in magnification view). Toluidine blue stain. Black scale bar shows $500 \mu \mathrm{m}$.

and that the composite of newly formed bone and $\beta$ TCP sufficiently restored the defect area (Fig 5d). Further, newly formed bone was seen in the micropores of remaining $\beta$-TCP. It should be highlighted that new bone formation in Fig. 5 did not depend on the presence of PRP. It should also be mentioned that toluidine blue metachromasia was seen in the superficial parts of bone in all groups but not in the micropores of groups using $\beta$-TCP granules.

At five weeks, the control site (Fig. 6a) showed dense bone trabeculae but the bone was thin. The Allograft-with-PRP group (Fig. 6b) showed newly formed bone with sufficient density, thickness and connection with the original bone. In groups with $\beta$ TCP granules, the TCP-with-PRP group (Fig. 6c) showed insufficient bone formation, whereas the Combined-with-PRP group (Fig. 6d) showed sufficient filling of the bone defect with dense bone trabeculae. For the two latter groups, a small amount of remnant $\beta$-TCP could be seen. It is noteworthy that the findings in the five-week groups with PRP were not different from those without PRP.

\section{Histomorphometric analyses}

1. Bone formation rate (BFR)

Table 1 presents the BFR results of each group. At one week and without PRP, the BFR results of the control, Allograft, TCP, and Combined groups were $3.8 \%, 12.1 \%, 4.8 \%$, and $4.3 \%$ respectively; with PRP, the BFR results were $3.4 \%, 5.7 \%, 7.6 \%$, and $6.9 \%$ respectively. Compared to the other groups, high BFR was seen in the Allograft group without PRP. Nonetheless, this high rate was not considered statistically significant compared with the BFR results of other test groups and the control at one week (Table 1).

At three weeks and with PRP, the BFR results of the control, Allograft, TCP, and Combined groups were $21.5 \%, 40.8 \%, 30.8 \%$, and $32.0 \%$ respectively; with PRP, the results were $20.8 \%, 41.2 \%, 24.2 \%$, and $41.3 \%$ respectively. Therefore, the BFR of the Allograft group without PRP was significantly higher than the control, and that of the Combined-with-PRP group was significantly higher than the control (Table 1, $\mathrm{P}<0.05$ ). Although not statistically 


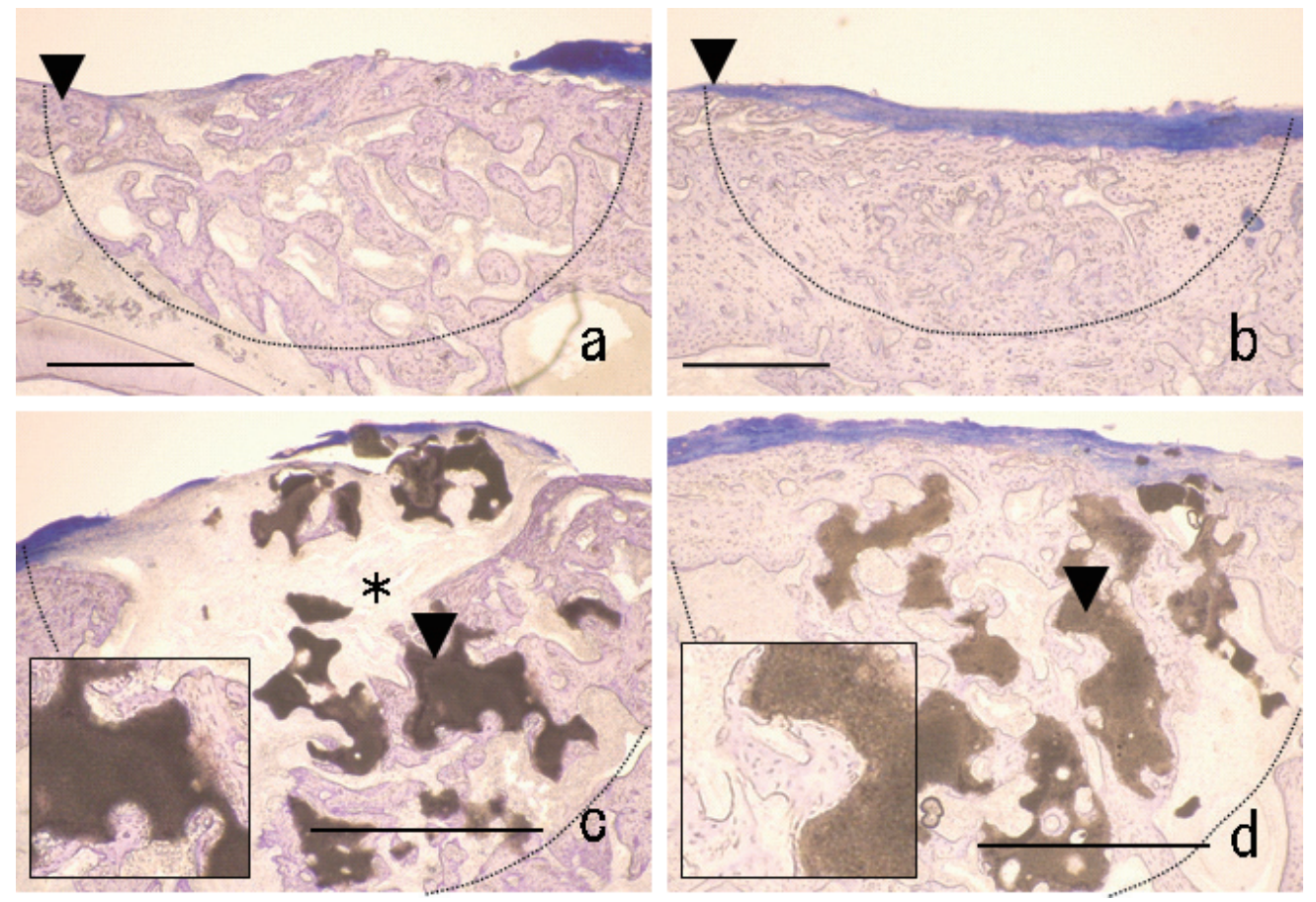

Fig. 5 Histological findings of graft materials $(b-d)$ and control site (a) without PRP in rat mandibular bone defects at three weeks after graft surgery. Measurement area is shown as a dotted line. (a) Control site without PRP: newly formed bone is thin and loose. Connection between new bone and original bone seems weak (arrowhead). (b) Allograft group without PRP: newly formed bone is woven and thick. Rigid connection between new bone and original bone is seen (arrowhead). (c) TCP group without PRP: newly formed bone is insufficient at center and upper part of defect $(*)$. Remnant $\beta$-TCP is seen (arrowhead). New bone is seen contacting the micropores of $\beta$-TCP (magnification view). (d) Combined group without PRP: comparatively good newly formed bone is seen uniformly in defect (dotted line). The composite of newly formed bone and $\beta$-TCP sufficiently restored the defect area. Remnant $\beta$ TCP well cooperated with newly formed bone (arrowhead). Newly formed bone in micropores of $\beta$-TCP (magnification view). Toluidine blue stain. Black scale bar shows $500 \mu \mathrm{m}$.

significant, the Combined-with-PRP group showed considerably higher BFR compared to that without PRP (Table 1, P=0.052).

At five weeks and without PRP, the BFR results of the control, Allograft, TCP, and Combined groups were $29.5 \%, 49.8 \%, 34.9 \%$, and $53.1 \%$ respectively; with PRP, the BFR results were $32.2 \%, 56.3 \%$, $41.3 \%$, and $55.5 \%$ respectively. Therefore, the BFR results of the Allograft and Combined groups were almost the same with or without PRP, and which were significantly higher than those of the control and TCP groups (Table $1, \mathrm{P}<0.05$ ).

\section{2. $\beta$-TCP absorption rate (TAR)}

Table 2 presents the TAR results of each group. For the TCP group, the TAR results were $77.2 \%$ at one week, $80.5 \%$ at three weeks, and $95.1 \%$ at five weeks without PRP; with PRP, the TAR results were $68.3 \%$ at one week, $86.9 \%$ at three weeks, and $94.7 \%$ at five weeks. For the Combined group, the TAR results were $47.5 \%$ at one week, $76.5 \%$ at three weeks, and 96.0\% at five weeks without PRP; with PRP, the TAR results were $46.8 \%$ at one week, $89.9 \%$ at three weeks, and $94.0 \%$ at five weeks. At one week, the TCP group with and without PRP showed higher TAR results than the Combined group (Table 2, $\mathrm{P}<0.05)$. At three weeks, the Combined-with-PRP group showed higher TAR than that without PRP (Table 2, $\mathrm{P}<0.05$ ).

3. Total rate of BFR and RTR (TBR)

Table 3 presents the TBR results of each group. For the TCP group, the TBR results were $27.6 \%$ at one week, $50.3 \%$ at three weeks, and $39.8 \%$ at five weeks without PRP; with PRP, the TBR results were $39.3 \%$ at one week, $37.3 \%$ at three weeks, and $46.6 \%$ at five weeks. For the Combined group, the TBR results were $56.8 \%$ at one week, $55.5 \%$ at three weeks, and $57.1 \%$ at five weeks without PRP; with PRP, the TBR results were $60.2 \%$ at one week, $51.4 \%$ at three 

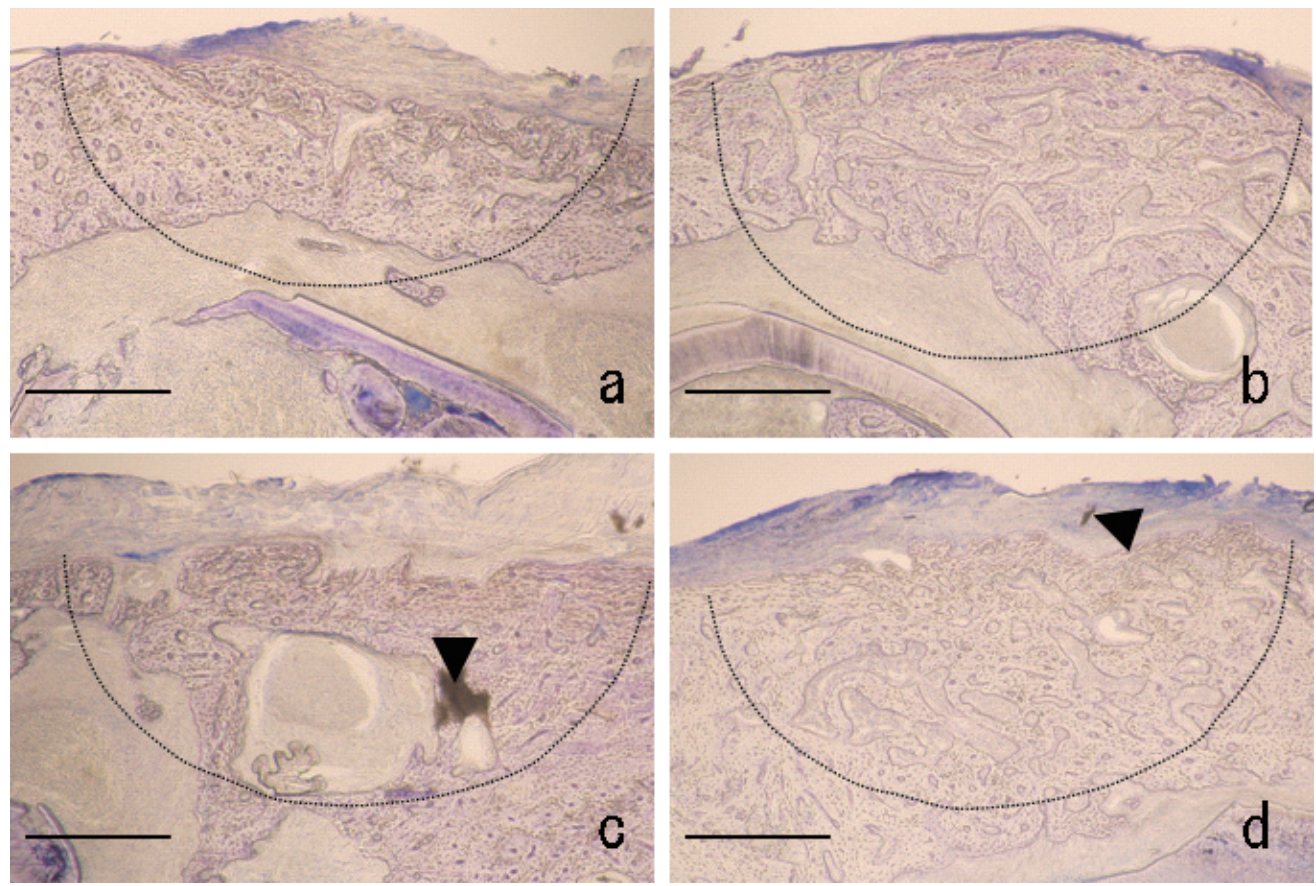

Fig. 6 Histological findings of graft materials $(b-d)$ and control site (a) with PRP in rat mandibular bone defects at five weeks after graft surgery. Measurement area is shown as a dotted line. (a) Control-with-PRP site: newly formed bone is thin and loose compared with the allogenic bone graft. (b) Allograft-with-PRP group: newly formed bone is thick and dense. New bone is rigidly connected with original bone. (c) TCP-with-PRP group: remnant $\beta$-TCP is seen (arrowhead). Newly formed bone around $\beta$-TCP is poor. (d) Combined-with-PRP group: newly formed bone fills defect area. A little remnant of $\beta$-TCP is seen (arrowhead). Bone connection between new bone and original bone is sufficient. Toluidine blue stain. Black scale bar shows $500 \mu \mathrm{m}$.

Table 1 Bone formation rate (BFR) (\%) results

\begin{tabular}{lcrrr}
\hline \multicolumn{1}{c}{ Group } & Control & Allograft & TCP & Combined \\
\hline 1 week & $3.8(3.8)$ & $12.1(5.2)$ & $4.8(3.7)$ & $4.3(0.7)$ \\
1 week with PRP & $3.4(3.4)$ & $5.7(2.4)$ & $30.8(5.9)$ & $6.9(2.0)$ \\
3 weeks & $21.5(4.7)$ & $* 40.8(6.3)$ & $41.2(15.4)$ & $32.0(5.2)$ \\
3 weeks with PRP & $20.8(5.6)$ & $* 49.8(6.8)$ & $34.9(4.8)$ & $* 41.3(3.6)$ \\
5 weeks & $29.5(7.1)$ & $* 56.3(5.2)$ & $41.3(5.4)$ & $* 53.1(9.4)$ \\
5 weeks with PRP & $32.2(4.1)$ & & $*$ \\
\hline
\end{tabular}

Data are presented as mean value (S.D.)

Table $2 \quad \beta$-TCP absorption rate (TAR) (\%) results

\begin{tabular}{lrr}
\hline \multicolumn{1}{c}{ Group } & \multicolumn{1}{c}{ TCP } & Combined \\
\hline 1 week & $* 77.2(4.6)$ & $47.5(4.9)$ \\
1 week with PRP & $* 68.3(13.1)$ & $46.8(4.2)$ \\
\hline 3 weeks & $80.5(4.7)$ & $76.5(6.5)$ \\
3 weeks with PRP & $86.9(4.7)$ & $* 89.9(1.7)$ \\
5 weeks & $95.1(1.7)$ & $96.0(1.2)$ \\
5 weeks with PRP & $94.7(1.6)$ & $94.0(4.2)$ \\
\hline
\end{tabular}

Data are presented as mean value (S.D.)
Table 3 Total of BFR and RTR (TBR) (\%) results

\begin{tabular}{lcc}
\hline \multicolumn{1}{c}{ Group } & TCP & Combined \\
\hline 1 week & $27.6(2.9)$ & $* 56.8(5.1)$ \\
1 week with PRP & $39.3(11.4)$ & $* 60.2(3.8)$ \\
3 weeks & $50.3(12.1)$ & $55.5(8.9)$ \\
3 weeks with PRP & $37.3(12.6)$ & $51.4(3.2)$ \\
5 weeks & $39.8(5.2)$ & $* 57.1(9.0)$ \\
5 weeks with PRP & $46.6(6.1)$ & $* 61.5(4.7)$ \\
\hline
\end{tabular}

Data are presented as mean value (S.D.) 
Table 4 Whole amount of $\beta$-TCP (\%) results

\begin{tabular}{lcc}
\hline \multicolumn{1}{c}{ Group } & TCP & Combined \\
\hline 1 week & $22.8(4.6)$ & $26.3(2.4)$ \\
1 week with PRP & $31.7(13.1)$ & $26.6(2.1)$ \\
\hline 3 weeks & $19.5(6.0)$ & $11.8(3.2)$ \\
3 weeks with PRP & $13.1(4.7)$ & $5.0(0.9)$ \\
5 weeks & $4.9(1.7)$ & $2.0(0.6)$ \\
5 weeks with PRP & $5.4(1.6)$ & $3.0(2.0)$ \\
\hline
\end{tabular}

Data are presented as mean value (S.D.)

weeks, and $61.5 \%$ at five weeks. At one and five weeks, the Combined group with or without PRP showed higher TBR results than the TCP group (Table 3, $\mathrm{P}<0.05$ ).

4. Whole amount of $\beta$-TCP (WTCP)

Table 4 presents the WTCP results of each group. For the TCP group, the WTCP results were $22.8 \%$ at one week, $19.5 \%$ at three weeks, and $4.9 \%$ at five weeks without PRP; with PRP, the WTCP results were $31.7 \%$ at one week, $13.1 \%$ at three weeks, and $5.4 \%$ at five weeks. For the Combined group, the WTCP results were $26.3 \%$ at one week, $11.8 \%$ at three weeks, and $2.0 \%$ at five weeks without PRP; with PRP, the WTCP results were $26.6 \%$ at one week, $5.0 \%$ at three weeks, and $3.0 \%$ at five weeks. There were no significant differences in WTCP results among the groups (Table 4); that is, the WTCP results were almost the same between the TCP and Combined groups at one, three, and five weeks.

\section{DISCUSSION}

Autogenous bone graft is the gold standard to restore or reconstruct bone defects. In oral and maxillofacial surgery, the iliac bone, tibia, mandibular symphysis and mandibular ramus are harvested ${ }^{9}$. To reduce or replace autogenous bone harvesting, it is necessary to develop a biocompatible bone substitute as a scaffold for bone regeneration. This study described the efficacy of highly pure porous $\beta$-TCP granules, OSferion ${ }^{\circledR}$, as a scaffold for bone regeneration of rat mandibular bone defects. This material was used in two ways, alone and combined with allogenic bone, at a 50:50 volume ratio. Although the bone defect was not of a critical size, there were apparent differences in bone formation between the control and the other test sites. In light of the findings obtained, the results were thus considered valid and acceptable to be used to evaluate the capabilities of allogenic bone and $\beta$-TCP granules for bone regeneration.

OSferion ${ }^{\circledR}$ has numerous interconnecting pathways similar to cancellous bone. On this ground, the material was expected to play the role of a bone regeneration scaffold similar to allogenic bone graft in the present study. However, the $\beta$-TCP granulesalone graft showed early absorption without sufficient bone regeneration. The $\beta$-TCP absorption rate (TAR) was clearly different between the TCP and Combined groups at one week. The TAR of TCP group was higher than that of the Combined group at one week. In the TCP group, almost $70 \%$ of $\beta$ TCP was absorbed without sufficient bone regeneration at one week. In other words, only $30 \%$ of $\beta$-TCP was left at this stage - in contrast to the almost $50 \%$ of $\beta$-TCP which remained in the Combined group. As both TCP and Combined groups showed increases in bone formation rate (BFR) at three and five weeks, the remaining $\beta$-TCP at one week could have indeed functioned as a scaffold for bone regeneration at these stages. According to the results obtained, the whole amount of $\beta$-TCP (WTCP) results in the TCP and Combined groups were almost the same at one week and in the subsequent stages. At this juncture, it should be mentioned that in the Combined group, the allogenic bone would have also fulfilled its pivotal role in bone regeneration. Consequently, the BFR of the Combined group was higher than that of the TCP group at five weeks.

These differences in TAR at one week and in BFR at five weeks affected the total rate of BFR and RTR (TBR). The TBR results were different between the TCP and Combined groups at one and five weeks, the early and late stages of bone regeneration. In both groups at one and five weeks, TBR was higher in the Combined group than in the TCP group. These differences were attributed to the higher TAR of the TCP group at one week and the higher BFR of the Combined group at five weeks. It is noteworthy that a higher TBR could mean that the graft material sufficiently restored the bone defect. The BFR of the Combined group was almost equivalent to that of the Allograft group. This result seemed to indicate that the absorption rate of $\beta$-TCP granules was reduced when they were combined with allogenic bone, thus enabling the $\beta$-TCP granules to effectively play the role of a bone regeneration scaffold equivalent to that played by an allogenic bone graft. In the same vein, Dong et $a l .{ }^{10)}$ reported that composites of $\beta$-TCP and bone marrow-derived osteoprogenitor cells showed good osteogenic activity. Therefore, $\beta$-TCP could be a good scaffold material for bone regeneration when used in combination with osteogenic materials. Osteogenic cells invade the micropores of $\beta$-TCP much more easily, thereby enabling bone formation in the micropores.

Although newly formed bone was observed, residues of $\beta$-TCP were seen at all stages in TCP 
and Combined groups. In oral surgery, a bone substitute is not merely a reconstruction material but that it is also part of the structure at recipient sites to place dental implants. Many studies have reported on slow absorption of bovine bone substitutes without adversely affecting the success rate of dental implants ${ }^{11-13)}$. However, it should be put into perspective that slow absorption of bone substitute materials could be an acceptable phenomenon for dental implants. Nonetheless, it is important that bone substitute materials be replaced by new bone over time. On a separate note, it is not necessary for the bone substitute material to be completely absorbed before implant placement. Nonetheless, it is important that bone regeneration should accompany the complete absorption of bone substitute material. Chasono et al. ${ }^{8}{ }^{8}$ reported that although the absorption phase of $\beta$-TCP granules was totally completed with favorable bone formation, a little remnant of this material could be observed. Further, Gaasbeek et al. ${ }^{1)}$ reported that clinically accepted remnants of $\beta$-TCP grafted for an osteotomy gap after high tibial osteotomy were observed from biopsy samples taken more than six months after graft surgery.

During the degradation phase of $\beta$-TCP, free $\beta$ TCP degradation particles and phagocytic cell accumulation were found around the newly formed bone - and this could negatively influence the osteointegration of dental implants inserted simultaneously with $\beta$-TCP ${ }^{2)}$. However, Oyake et al. ${ }^{14)}$ observed bone formation between $\beta$-TCP particles applied around a titanium rod in rabbits and reported on rigid pull-out strength of the titanium rod and delayed absorption of $\beta$-TCP. Similarly, Cerasorb $^{\circledR}$, a resorbable, fully synthetic, pure-phase $\beta$-TCP matrix with interconnecting porosity, showed effective replacement by bone tissue and gradual absorption of $\beta$-TCP in human sinus floor augmentation before implant surgery ${ }^{7}$. Although the influence of remnant $\beta$-TCP on bone tissue remains unknown, the residues seen in this study could be an acceptable consequence of $\beta$-TCP playing a favorable role as a bone regeneration scaffold.

Significant association between PRP and bone formation was not observed in the present study. Nonetheless, in the Combined group at three weeks, PRP accelerated the absorption of $\beta$-TCP, consequently causing the BFR of the Combined-withPRP group to be considerably higher - although not statistically significant - than that of the same group without PRP at three weeks. This result seemed to suggest that PRP favorably promoted early bone replacement from $\beta$-TCP when used with an allogenic bone graft. Presently, there is a lack of scientific knowledge on the effectiveness of PRP in osteoblast-like cell proliferation and bone regeneration. On one hand, some studies have reported that $\mathrm{PRP}$ had no effect on bone-implant contact in one-stage sinus graft and simultaneous implant placement ${ }^{15-17)}$. On the other hand, many recent reports have claimed that PRP promoted osteoblast cell proliferation ${ }^{18-20)}$. When used in combination with $\beta$-TCP alone, Wiltfang et $a l .{ }^{5}$ ) reported that $\mathrm{PRP}$ did not accelerate the absorption of $\beta$-TCP for sinus floor augmentation and neither did it enhance bone regeneration. In consideration of the aforementioned reports, it could be said that PRP - when used with an osteogenic graft material like autogenous bone - could enhance cell proliferation and bone substitute material absorption, thereby inducing early bone regeneration.

One key practical benefit of $\beta$-TCP is its ease of use in oral surgery. Therefore, it is highly likely that the biocompatibility and biodegradability of this material be further evaluated for its applicability in bone regeneration. This study showed that OSferion $^{\circledR}$, when used with an allogenic bone graft, was an effective bone substitute scaffold for bone regeneration because its early absorption without bone regeneration was reduced. Based on the results of this study, $\beta$-TCP augers well as a new scaffold candidate for bone regeneration when used in combination with osteogenic materials, such as autogenous bone and osteoprogenitor cells, in oral and maxillofacial bone graft surgery.

\section{REFERENCES}

1) Gaasbeek RD, Toonen HG, van Heerwaarden RJ, Buma P. Mechanism of bone incorporation of $\beta$-TCP bone substitute in open wedge tibial osteotomy in patients. Biomater 2005; 26: 6713-6719.

2) Merten HA, Wiltfang J, Grohmann U, Hoenig JF. Intraindividual comparative animal study of $\alpha$ - and $\beta$-Tricalcium phosphate degradation in conjunction with simultaneous insertion of dental implants. J Craniofac Surg 2001; 12: 59-68.

3) Kurashina K, Kurita H, Wu Q, Ohtsuka A, Kobayashi H. Ectopic osteogenesis with biphasic ceramics of hydroxyapatite and tricalcium phosphate in rabbits. Biomater 2002; 23: 407-412.

4) De Groot K. Bioceramics consisting of calcium phosphate salts. Biomater 1980; 1: 47-50.

5) Wiltfang J, Schlegel KA, Schultze-Mosgau S, Nkenke E, Zimmermann R, Kessler P. Sinus floor augmentation with $\beta$-tricalcium phosphate $(\beta$-TCP): does platelet-rich plasma promote its osseous integration and degradation? Clin Oral Impl Res 2003; 14: 213-218.

6) Zerbo IR, Zijderveld SA, de Boer A, Bronckers ALJJ, Lange $\mathrm{G}$, ten Bruggenkate CM, Burger EH. Histomorphometry of human sinus floor augmentation using a porous $\beta$-tricalcium phosphate: a prospective study. Clin Oral Impl Res 2004; 15: 724-732.

7) Suba Z, Takacs D, Matusovitis D, Barabas J, Fazekas A, Szabo G. Maxillary sinus floor grafting 
with $\beta$-tricalcium phosphate in humans: density and microarchitecture of the newly formed bone. Clin Oral Impl Res 2006; 17: 102-108.

8) Chazono M, Tanaka T, Komaki H, Fujii K. Bone formation and bioresorption after implantation of injectable $\beta$-tricalcium phosphate granuleshyaluronate complex in rabbit bone defects. J Biomed Mater Res 2004; 70A: 532-549.

9) Garg AK. Review of bone-grafting materials. In: Bone biology, harvesting, grafting for dental implants: rationale and clinical applications, 1st ed, Garg AK (ed.), Quintessence, Chicago, 2004, pp.2156.

10) Dong J, Uemura T, Shirasaki Y, Tateishi T. Promotion of bone formation using highly pure porous $\beta$-TCP combined with bone marrow-derived osteoprogenitor cells. Biomater 2002; 23: 4493-4502.

11) Schlegel KA, Donath K. Bio-Oss - a resorbable bone substitute? Int J Biomat Med Implants 1998; 8: 201-209.

12) Tadjoedin ES, de Lange GL, Bronckers AL, Lyaruu DM, Burger EH. Deproteinized cancellous bovine bone (Bio-Oss) as bone substitute for sinus floor elevation. J Clin Periodontol 2003; 30: 261-270.

13) Sartori S, Silvestri M, Forni F, Cornaglia IA, Tesei $\mathrm{P}$, Cattaneo V. Ten-year follow-up in a maxillary sinus augmentation using anorganic bovine bone (Bio-Oss). A case report with histomorphometric evaluation. Clin Oral Impl Res 2003; 14: 369-372.

14) Oyake Y, Beppu M, Ishii S, Takagi M, Takashi M. Intramedullary anchoring strength of titanium rod with mixed $\beta$-tricalcium phosphate and fibrin adhesive. J Orthop Sci 2002; 7: 123-130.

15) Klongnoi B, Rupprecht S, Kessler P, Thorwarth M, Wiltfang J, Schlegel KA. Influence of platelet-rich plasma on a bioglass and autogenous bone in sinus augmentation. An explorative study. Clin Oral Impl Res 2006; 17: 312-320.

16) Klongnoi B, Rupprecht S, Kessler P, Zimmermann R, Thorwarth M, Pongsiri S, Neukam FW, Wiltfang J, Schlegel KA. Lack of beneficial effects of plateletrich plasma on sinus augmentation using a fluorohydroxyapatite or autogenous bone: an explorative study. J Clin Periodontol 2006; 33: 500-509.

17) Schlegel KA, Thorwarth M, Plesinac A, Wiltfang J, Rupprecht S. Expression of bone matrix proteins during the osseus healing of topical conditioned implants: an experimental study. Clin Oral Impl Res 2006; 17: 666-672.

18) Ferreira CF, Gomes MCC, Filho JS, Granjeiro JM, Oliveira Simões CM, Magini RS. Platelet-rich plasma influence on human osteoblasts growth. Clin Oral Impl Res 2005; 16: 456-460.

19) Graziani F, Ivanovski S, Cei S, Ducci F, Tonetti M, Gabriele M. The in vitro effect of different PRP concentrations on osteoblasts and fibroblasts. Clin Oral Impl Res 2006; 17: 212-219.

20) Gruber R, Varga F, Fischer MB, Watzek G. Platelet stimulate proliferation of bone cells: involvement of platelet-derived growth factor, microparticles and membranes. Clin Oral Impl Res 2002; 13: 529-535. 